\title{
MULTIPOLE RADIATION IN QUANTUM DOMAIN
}

\author{
Alexander S. Shumovsky \\ Physics Department, Bilkent University \\ Bilkent, 06800 Ankara, Turkey \\ M. Ali Can \\ Physics Department, Bilkent University \\ Bilkent, 06800 Ankara, Turkey
}

Öney Soykal

Physics Department, Bilkent University

Bilkent, 06800 Ankara, Turkey

shumo@ fen.bilkent.edu.tr

\begin{abstract}
We review recent results based on the application of spherical wave representation to description of quantum properties of multipole radiation generated by atomic transitions. In particular, the angular momentum Angular momentum of photons including the angular momentum entanglement, the quantum phase of photons, and the spatial properties of polarization are discussed.
\end{abstract}

Keywords: Angular momentum, atoms, entanglement, polarization, quantum phase, qutrit, spherical waves of photons.

\section{Introduction}

It is well known since the end of XIX century that the time-varying classical electromagnetic (EM) field can be expanded in vector spherical waves and that this representation is convenient for electromagnetic boundary-value problems possessing spherical symmetry and for the discussion of multipole radiation from a local sources (e.g., see [1,2]). Since both plane and spherical waves form complete sets of orthonormal functions, they are equivalent, so that the use of either representation of classical electromagnetic radiation is caused by the usability reasons. 
The underlying motive for consideration of quantum EM radiation in terms of spherical waves of photons is the fact that the atomic and molecular transitions create the multipole photons, in other words, the photons with given angular momentum and parity rather than plane photons specified by the linear momentum and polarization polarization $[3,4]$.

It should be stressed that, unlike the classical domain, the plane and spherical wave representations of photons are not completely equivalent. The point is that the vector potential of classical EM field is defined in the three-dimensional Euclidian space $\mathcal{R}^{3}$, while the operator vector potential of quantum EM radiation is defined in the space

$$
\mathcal{H}=\mathcal{R}^{3} \otimes \mathcal{H}_{p h},
$$

where $\mathcal{H}_{p h}$ denotes the Hilbert space of photons. According to Wigner's approach [5], the general properties of a quantum mechanical system are specified by the dynamic symmetry of the corresponding Hilbert space. The Hilbert spaces of plane and spherical photons have different symmetry properties. Viz, the former manifests the $S O(2)$ symmetry caused by invariance with respect to rotations in $x y$-plane whose positive normal coincides with direction of propagation $\vec{k} / k$. In turn, the latter has the $S U(2)$ symmetry agreed upon the invariance with respect to rotations in three dimensions about a local source (say, atom or molecule).

In particular, the symmetry reasons imply the different sets of quantum numbers, specifying the photons in the two representations $[3,4,6]$. Any photon in the plane wave representation (PWR) is specified by given energy, linear momentum, and polarization. In turn, a photon in the spherical wave representation (SWR) has given energy, angular momentum Angular momentum, and parity that corresponds to the type of radiation, either electric or magnetic.

SWR was introduced by Heitler [7] and then discussed in his famous monograph [6]. Unfortunately, the most of books on quantum optics leave this representation aside, so that it is discussed in very few monographs (e.g., see $[3,4])$. Meanwhile, a number of modern experiments with trapped atoms interacting with photons corresponds to the interatomic distances that are much shorter than the wave length $[8,9]$. It should be stressed that the difference between the properties of photons in PWR and SWR is particularly strong just in the near and intermediate zones.

Another reason to use SWR is connected with the problem of use of the angular momentum (AM) of photons in quantum information processing that has attracted recently a great deal of interest $[10,11,12,13,14]$. In the usual treatment, AM of photons is discussed in terms of PWR [10, 15]. Unfortunately, this representation leads to the wrong commutation relations for spin spin components as well as for orbital angular momentum (OAM) components $[10,16]$. 
The great importance of true commutation relations in quantum mechanics is obvious to everyone. In fact, just existence of nontrivial commutation relations for observables is the distinguishing feature of quantum mechanics in comparison with classical physics. In particular, the commutation relations are responsible for the quantum noise that influences the precision of measurements in quantum domain. Unlike PWR, the use of SWR leads to the true commutation relations for spin and OAM operators [17].

Besides that, SWR can be used to define the inherent quantum phase phase of photons $[18,19,20,21]$, to describe the quantum properties of polarization $[22,23]$, and to discuss emission of photons with high AM [24].

The main aim of present course is to give a brief review of SWR, its application, and recent results related to the problem of quantum communications and information processing.

The course is arranged as follows. In Sec. I we introduce SWR and discuss its general properties. In Sec. II we consider representation of AM in SWR. In particular, we examine AM entanglement entanglement of two photons emitted by a cascade decay of an electric quadrupole transition. In Sec. III inherent quantum phase of multipole photons is discussed. In Sec. IV we consider the polarization of quantum multipole radiation. Finally in Sec. V we briefly summarize the results and discuss their applications.

\section{Quantization of Multipole Radiation}

Following [6], let us construct a representation of photons with given AM and parity. This means that we have to represent the vector potential in terms of a superposition of states with given AM and parity.

As for any other particle, AM of a photon consists of the spin and OAM contributions

$$
\vec{J}=\vec{S}+\vec{L}
$$

where $\vec{S}$ and $\vec{L}$ denote the spin spin and OAM, respectively. Since rest mass of photons is equal to zero, the spin is defined to be the minimum possible value of AM. From the atomic spectroscopy we know that minimum $j=1$ (in the units of $\hbar$ ). Hence, spin of a photon is $s=1$. Nevertheless, the requirement of Poincaré invariance on the light cone allows only two spin degrees of freedom.

The eigenfunctions of the operators $J_{z}$ and $\vec{J}^{2}$ are the vector spherical harmonics $[2,3,25]$

$$
\vec{J}^{2} \vec{Y}_{j \ell m}=j(j+1) \vec{Y}_{j \ell m}, \quad J_{z} \vec{Y}_{j \ell m}=m \vec{Y}_{j \ell m} .
$$

The eigenstates of spin 1 are the columns

$$
\vec{\epsilon}_{+}=\left(\begin{array}{c}
1 \\
0 \\
0
\end{array}\right), \quad \vec{\epsilon}_{0}=\left(\begin{array}{l}
0 \\
1 \\
0
\end{array}\right), \quad \vec{\epsilon}_{-}=\left(\begin{array}{l}
0 \\
0 \\
1
\end{array}\right),
$$


that can be associated with the base vectors in $\mathcal{R}^{3}$ as follows

$$
\vec{\epsilon}_{ \pm}=\mp \frac{\vec{e}_{x} \pm i \vec{e}_{y}}{\sqrt{2}}, \quad \vec{\epsilon}_{0}=\vec{e}_{z} .
$$

In fact, the vectors (4) form the so-called helicity basis [2,26]. In particular, the vectors $\vec{\epsilon}_{ \pm}$can be associated with unit vectors of polarization with either positive or negative helicity. Let us stress that quantum electrodynamics interprets the polarization as a given spin state of photons [3].

In turn, the eigenstates of quantum mechanical OAM operator $-i(\vec{r} \times \vec{\nabla})$ are the spherical harmonics $Y_{\ell m}(\vec{k} / k)$. Thus, the vector spherical harmonics (3) can be constructed as the linear combinations of spin states and spherical harmonics

$$
\vec{Y}_{j \ell m}=\sum_{\mu}\langle 1 \ell \mu, m-\mu \mid j m\rangle \vec{\epsilon}_{\mu} Y_{\ell, m-\mu},
$$

where $\langle\cdots \mid \cdots\rangle$ denotes the Clebsch-Gordon coefficients of vector addition of spin spin and OAM. Taking into account the properties of Clebsch-Gordon coefficients [27], it is easy to conclude that the quantum numbers $j$ and $\ell$ connected in the following way

$$
j=\ell+1, \ell,|\ell-1| .
$$

Thus, for each value of $\mathrm{AM} j$, there are three different states specified by the vector spherical harmonics (5) under the condition (6).

Since under inversion vector $\vec{\epsilon}_{\mu}$ changes sign and function $Y_{\ell, m-\mu}$ is multiplied by $(-1)^{\ell}$, the vector spherical harmonics have given parity $(-1)^{\ell+1}$. Thus, the functions $\vec{Y}_{j j m}$ have the parity $(-1)^{j+1}$, while the parity of functions $\vec{Y}_{j, j \pm 1, m}$ is $(-1)^{j}$.

The vector spherical harmonics (5) form a complete orthonormal set of functions:

$$
\int_{0}^{2 \pi} d \phi \int_{0}^{\pi} \vec{Y}_{j \ell m}^{+} \cdot \vec{Y}_{j^{\prime} \ell^{\prime} m^{\prime}} \sin \theta d \theta=\delta_{j j^{\prime}} \delta_{\ell \ell^{\prime}} \delta_{m m^{\prime}}
$$

It is seen that $(\vec{k} / k) \cdot \vec{Y}_{j j m}(\vec{k} / k)=0$. This function $\vec{Y}_{j j m}$ is usually cold the transversal vector spherical harmonics of magnetic type. Another transversal function can be constructed as a combination of the functions with $\ell=j \pm 1$

$$
\vec{Y}_{j m}^{E} \equiv \frac{1}{\sqrt{2 j+1}}\left(\sqrt{j} \vec{Y}_{j, j+1, m}+\sqrt{j+1} \vec{Y}_{j, j-1, m}\right),
$$

which is called the transversal spherical harmonics of electric type. It is seen that $\vec{Y}_{j j m}$ and $\vec{Y}_{j m}^{E}$ are mutually orthogonal for the same $\vec{k} / k$. 
The functions with $\ell=j \pm 1$ can also be used to construct the longitudinal vector spherical function

$$
\vec{Y}_{j m}^{L}=-\sqrt{\frac{j+1}{2 j+1}} \vec{Y}_{j, j+1, m}+\sqrt{\frac{j}{2 j+1}} \vec{Y}_{j, j-1, m},
$$

which is orthogonal to both $\vec{Y}_{j j m}$ and $\vec{Y}_{j m}^{E}$. Nevertheless, this longitudinal function should be discarded because the Poincaré invariance on the light cone.

Thus, the states of the field with given AM and parity can be obtained by expansion of vector potential over the transversal vector spherical harmonics. Taking into account the expansion [2]

$$
e^{i\left(\vec{k} \cdot \vec{r}-\omega_{k} t\right)}=4 \pi \sum_{\ell, m}(i)^{\ell} j_{\ell}(k r) Y_{\ell m}^{*}(\vec{k} / k) Y_{\ell m}(\vec{k} / k) e^{-i \omega_{k} c},
$$

where $j_{\ell}(k r)$ denotes the spherical Bessel functions, we can conclude that the positive-frequency part of the vector potential has the form

$$
\begin{aligned}
\vec{A}_{M k j m} & =N_{M j} \vec{Y}_{j j m} a_{M k j m} \\
\vec{A}_{E k j m} & =N_{E}\left[\sqrt{j} j_{j+1}(k r) \vec{Y}_{j, j+1, m}-\sqrt{j+1} j_{j-1}(k r) \vec{Y}_{j, j-1, m}\right] a_{E k(j h)}
\end{aligned}
$$

in the case of parity $(-1)^{j+1}$ and $(-1)^{j}$, respectively. Here $N_{\lambda}$ denotes the normalization factor. In order to have vector potential with discrete values of $k$, the right-hand sizes in (9) and (10) should be defined inside an ideal spherical cavity of big radius $R$. Then, the spectrum is defined by the roots of equation

$$
j_{\ell}(k R)=0 .
$$

In this case, it is convenient to renormalize the spherical Bessel functions by the condition

$$
\forall \ell \quad \int_{0}^{R} j_{\ell}(k r) j_{\ell}\left(k^{\prime} r\right) r^{2} d r=\frac{4 \pi R^{3}}{3} \delta_{k k^{\prime}} .
$$

In Eqs. (9) and (10), the complex amplitudes $a_{\lambda k j m}$ specify the amount of the corresponding multipole field. The harmonic time dependence is usually included into these amplitudes. In classical electrodynamics, the amplitudes $a_{\lambda k j m}$ are determined by the properties of the source of radiation (harmonically varying current or intrinsic magnetization) [2]. Within the quantum picture, the amplitudes $a_{\lambda k j m}$ are supposed to be the annihilation operators of multipole photons $[6,25]$ that obey the commutation relations

$$
\left[a_{\lambda k j m}, a_{\lambda^{\prime} k^{\prime} j^{\prime} m^{\prime}}^{+}\right]=\delta_{\lambda \lambda^{\prime}} \delta_{k k^{\prime}} \delta_{j j^{\prime}} \delta_{m m^{\prime}} .
$$

Hence, they form a representation of the Weyl-Heisenberg algebra of multipole photons. In this case, (9) and (2) should be considered as the positive frequency 
parts of the operator vector potential of the magnetic-type radiation (with $\lambda=$ $M$ and parity $(-1)^{j+1}$ ) and of the electric-type radiation (with $\lambda=E$ and parity $\left.(-1)^{j}\right)$, respectively.

Hereafter, we consider expressions (9) and (10) as the quantum operators.

Let us now note that the operators (9) and (10) can be represented in $\mathcal{R}^{3}$ as follows

$$
\vec{A}_{\lambda k j m}=\sum_{\mu}(-1)^{\mu} \epsilon_{-\mu} \mathcal{A}_{\lambda k j m \mu} a_{\lambda k j m}
$$

where $\mathcal{A}_{\lambda k j m \mu}$ denotes the mode function of the multipole field. By construction, this function obey the homogeneous Helmholtz wave equation

$$
\nabla^{2} \mathcal{A}_{\lambda k j m \mu}+\omega_{k}^{2} \mathcal{A}_{\lambda k j m \mu}=0 .
$$

In fact, the vector $\overrightarrow{\mathcal{A}}_{\lambda k j m}$ can be considered as a function from $\mathcal{R}^{3}$ to the Hilbert space $\mathcal{H}$ of complex linear functions on $\mathcal{R}^{3}$ in (1). The operators (9) and (10) obey the same wave equations but assumes values in the Hilbert space $\mathcal{H} \times \mathcal{H}$, where the second factor $\mathcal{H}$ comes from the spin states.

In view of the wave equation, the mode functions $\overrightarrow{\mathcal{A}}_{\lambda k j m}$ can be interpreted as the wave functions of multipole photons [3].

It should be emphasized that under rotations the vector spherical functions are transformed along an irreducible representations of the $\mathrm{O}^{+}(3)$ group. Thus, they are the irreducible tensors of rank $j$ rather than vectors.

It is useful to show that the operators (9) and (10) are invariant with respect to the $S U(2)$ group. Consider first the electric-type multipole radiation and introduce an auxiliary operator

$$
\overrightarrow{\mathbf{A}}_{\ell}(\vec{r} / r)=\sum_{\mu, m} Y_{1 \mu} Y_{\ell m} \vec{\epsilon}_{\mu} \otimes a_{\ell m}
$$

For simplicity, we drop here all other indexes. Because rotations do not influence the radial dependence in (10) provided by the spherical Bessel functions, the auxiliary function (12) depends only on the direction $\vec{r} / r$ in $\mathcal{R}^{3}$.

Let $\varphi$ be an arbitrary transformation belonging to the $S U(2)$ group. Then [28]

$$
\begin{aligned}
\overrightarrow{\mathbf{A}}_{\ell}(\varphi \vec{r} / r) & =\sum_{\mu, m} Y_{1 \mu}(\varphi \vec{r} / r) Y_{\ell m}(\varphi \vec{r} / r) \\
& =\sum_{\mu, \mu^{\prime}} \sum_{m, m^{\prime}} Y_{1 \mu^{\prime}}(\vec{r} / r) \varphi_{\mu \mu^{\prime}} Y_{\ell m^{\prime}}(\vec{r} / r) \vec{\epsilon}_{\mu} \otimes \varphi_{m m^{\prime}} a_{\ell m} \\
& =\sum_{\mu, \mu^{\prime}} \sum_{m, m^{\prime}} Y_{1 \mu^{\prime}}(\vec{r} / r) Y_{\ell m^{\prime}}(\vec{r} / r)\left[\varphi_{\mu \mu^{\prime}} \vec{\epsilon}_{\mu}\right] \otimes\left[\varphi a_{\ell m}\right] \\
& =\sum_{m u^{\prime}, m^{\prime}} Y_{1 \mu^{\prime}}(\vec{r} / r) Y_{\ell m^{\prime}}(\vec{r} / r)\left[\varphi \vec{\epsilon}_{\mu^{\prime}}\right] \otimes\left[\varphi a_{\ell m}\right]=\varphi \overrightarrow{\mathbf{A}}_{\ell}(\vec{r} / r) .
\end{aligned}
$$


Thus, the auxiliary operator (12) is invariant with respect to the $S U(2)$ group.

Since the spherical harmonics form a basis of an irreducible representation $\mathbf{M}_{\ell}$ of the $S U(2)$ group, the product $Y_{1 \mu} Y_{\ell m}$ in (12) form a basis of

$$
\mathbf{M}_{1} \otimes \mathbf{M}_{\ell}=\mathbf{M}_{\ell-1} \oplus \mathbf{M}_{\ell} \oplus \mathbf{M}_{\ell+1} .
$$

The operator (10) is defined just in (13).

Let $\left(Y_{1 \mu} Y_{\ell m}\right)_{s}(s=\ell, \ell \pm 1)$ be the component (projection) of $Y_{1 \mu} Y_{\ell m}$ in $\mathbf{M}_{s}$. Then the vector operator

$$
\overrightarrow{\mathbf{A}}_{\ell s}=\sum_{\mu, m}\left(Y_{1 \mu} Y_{\ell m}\right)_{s} \vec{\epsilon}_{\mu} \otimes a_{\ell m}
$$

is also invariant with respect to the $S U(2)$ group. This implies the invariance of (10), because rotations do not influence the radial dependence. The $S U(2)$ invariance of (9) can be proven in the same way.

\section{Angular Momentum of Multipole Photons}

A classical distribution of electromagnetic field in vacuum carries AM of the form

$$
\vec{J}=\frac{1}{4 \pi c} \int \vec{r} \times(\vec{E} \times \vec{B}) d^{3} r
$$

where

$$
\vec{E}=\frac{1}{c} \frac{\partial \vec{A}}{\partial t}, \quad \vec{B}=\vec{\nabla} \times \vec{A}
$$

are the electric and magnetic fields. For the fields produced a finite time in the past and so localized to a finite region, this expression can be rewritten in the form

$$
\vec{J}=\frac{1}{4 \pi c} \int\left[\vec{E} \times \vec{A}+\sum_{\mu} E_{\mu}(\vec{r} \times \vec{\nabla}) B_{\mu}\right] d^{3} r .
$$

The first term is usually identified with the spin contribution, while the second term represents OAM because of the presence of the quantum mechanical angular momentum Angular momentum operator $-i(\vec{r} \times \vec{\nabla})$ [15]. Let us stress that Eq. (15) is obtained within the classical picture, so that the use of the notions spin and OAM has here a conditional meaning.

Within the quantum domain, both terms in the right-hand side of (15) are represented by the bilinear forms in the photon operators (11).

Consider first PWR, when the operator vector potential has the form

$$
\vec{A}(\vec{r})=N \sum_{\mu= \pm} \sum_{k} \vec{\epsilon}_{k \mu}\left(e^{i \vec{k} \cdot \vec{r}} a_{k \mu}+\text { H.c. }\right)
$$


because the third direction $\epsilon_{k 0}$ is forbidden in this case [15]. Averaging the first term in the right-hand side of (15) over time to eliminate the rapidly oscillation terms with $a^{2}$ and $\left(a^{+}\right)^{2}$ and changing summation over $k$ by integration, we get for the spin spin operator

$$
\vec{S}=\frac{N^{2}}{2 \pi c} \int \frac{d^{3} k^{3}}{2 \pi} \vec{k}\left(a_{k+}^{+} a_{k+}-a_{k-}^{+} a_{k-}\right) .
$$

Thus, the components of "spin" (16) obey the commutation relations $[10,16]$ :

$$
\left[S_{\alpha}, S_{\beta}\right]=0, \quad \alpha, \beta=x, y, z .
$$

Employing the same procedure to the second (OAM) term in (15) then gives the following commutation relations

$$
\left[L_{\alpha}, L_{\beta}\right]=i \epsilon_{\alpha \beta \gamma}\left(L_{\gamma}+S_{\gamma}\right), \quad\left[L_{\alpha}, S_{\beta}\right]=i \epsilon_{\alpha \beta \gamma} S_{\gamma},
$$

so that neither spin nor OAM obey the true commutation relations. In fact, the definition (2) assumes the structure $S U(2) \times S U(2)$ of the total AM. At the same time, the above commutation relations correspond to the structure $\mathbf{A} \times S U(2)$, where $\mathbf{A}$ denotes the Abelian group of translations caused by the $S O(2)$ symmetry of the Hilbert space.

The use of SWA leads to a different result. According to the results of Sec. II, the total AM (2) has the structure $S U(2) \times S U(2)$ by construction.

Consider first a single-mode photon emitted by an electric dipole (E1) transition in a two-level atom two-level atom located at the center of an ideal spherical cavity. Let us stress that E1 photons represent the most frequently encountered type of EM radiation in visible and IR regions. If AM of the excited atomic state is $j=1$, then this state is triple degenerated with respect to the quantum number $m=0, \pm 1$. The Jaynes-Cummings Hamiltonian Hamiltonian of such a system has the form [18]

$$
\begin{aligned}
H & =H_{0}+H_{i n t}, \\
H_{0} & =\sum_{m}\left(\omega a_{m}^{+} a_{m}+\omega_{0} R_{m m}\right), \\
H_{i n t} & =\gamma \sum_{m}\left(R_{m g} a_{m}+a_{m}^{+} R_{g m}\right) .
\end{aligned}
$$

Here $\omega$ is the cavity mode frequency, $\omega_{0}$ is the atomic transition frequency, $\gamma$ is the coupling constant, $a_{m}, a_{m}^{+}$are the E1 photon operators (11), and $R$ denotes the atomic operators:

$$
R_{m m^{\prime}}=|j=1, m\rangle\left\langle j=1, m\left|, \quad R_{m g}=\right| j=1, m\right\rangle\left\langle j^{\prime}=0,0\right| .
$$

Since the angular momentum is conserved in the atom-photon interaction [4], the total angular momentum

$$
\overrightarrow{\mathcal{J}}=\vec{J}^{(a)}+\vec{J}^{(p h)}
$$


should be an integral of motion with the Hamiltonian (18). It is clear that the photon, created by the atom, takes away AM of the excited atomic state. The latter is specified by the operators

$$
\begin{array}{r}
J_{x}^{(a)}=\frac{1}{\sqrt{2}}\left(R_{0+}+R_{0-}+H . c .\right), \quad J_{y}^{(a)}=\frac{1}{\sqrt{2}}\left(R_{0+}-R_{-0}-H . c .\right), \\
J_{z}^{(a)}=R_{++}-R_{--},
\end{array}
$$

that obey the commutation relations

$$
\left[J_{\alpha}^{(a)}, J_{\beta}^{(a)}\right]=i \epsilon_{\alpha \beta \kappa} J_{\kappa}^{(a)}, \quad \alpha, \beta, \kappa=x, y, z .
$$

It is now a straightforward matter to arrive at conclusion that the photon operator, complementing (20) with respect to the integral of motion with the Hamiltonian (18), has the components

$$
\begin{array}{r}
J_{x}^{(p h)}=\frac{1}{\sqrt{2}}\left\{a_{0}^{+}\left(a_{+}+a_{-}\right)+H . c .\right\}, \\
J_{y}^{(p h)}=\frac{i}{\sqrt{2}}\left\{a_{0}^{+}\left(a_{+}-a_{-}\right)-H . c .\right\}, \\
J_{z}^{(p h)}=a_{+}^{+} a_{+}-a_{-}^{+} a_{-} .
\end{array}
$$

It follows from (11) that the operators (22) obey the same commutation relations as (21), that are the true commutation relations for the components of AM operator. By construction, the operators (22) define AM carried away by the photon from the atom. Thus, the use of SWR leads to the true commutation relations for $\mathrm{AM}$ of photons.

Let us stress the principle difference in the operator structure of Eqs. (15) and (22). In the former, the symbols \pm denote the circular polarization polarization, while in the latter, the subscripts $m=0, \pm 1$ correspond to the projection of angular momentum Angular momentum $j=1$ on the quantization axis.

Assume that the atom emits E1 photon with given $m$. Then, for the mean values of AM operators (21) we get

$$
\forall m \quad\left\langle 1_{m}\left|J_{x, y}^{(p h)}\right| 1_{m}\right\rangle=0, \quad\left\langle 1_{m}\left|J_{z}^{(p h)}\right| 1_{m}\right\rangle=m .
$$

In turn, the variances are

$$
\left\langle 1_{m}\left|\left(\Delta J_{x, y}^{(p h)}\right)^{2}\right| 1_{m}\right\rangle= \begin{cases}\frac{1}{2}, & \text { at } m= \pm 1 \\ 1, & \text { at } m=0\end{cases}
$$

and

$$
\left\langle 1_{m}\left|\left(\Delta J_{z}^{(p h)}\right)^{2}\right| 1_{m}\right\rangle=|m| .
$$


Thus, the Fock number state of E1 photon manifests strong quantum fluctuations of AM, and the quantum fluctuations of AM in the state with $m=0$ are stronger than those in the states with $m= \pm 1$.

Eqs. (21) can be used to specify mean values and variances of AM of many E1 photons as well. Assume for example that the local source emits E1 photons in coherent state coherent state $\left|\alpha_{m}\right\rangle$ with given $m$. Then

$$
\left\langle\alpha_{m}\left|J_{x, y}^{(p h)}\right| \alpha_{m}\right\rangle=0, \quad\left\langle\alpha_{m}\left|J_{z}^{(p h)}\right| \alpha_{m}\right\rangle=m\left|\alpha_{m}\right|^{2}
$$

and

$$
\begin{array}{r}
\left\langle\alpha_{m}\left|\left(\Delta J_{x, y}^{(p h)}\right)^{2}\right| \alpha_{m}\right\rangle= \begin{cases}\frac{1}{2}\left|\alpha_{ \pm}\right|^{2}, & m= \pm 1 \\
\left|\alpha_{0}\right|^{2}, & m=0\end{cases} \\
\left\langle\alpha_{m}\left|\left(\Delta J_{z}^{(p h)}\right)^{2}\right| \alpha_{m}\right\rangle=|m|\left|\alpha_{m}\right|^{2} .
\end{array}
$$

In PWR, the physical quantities related to the operators $J_{x, y}^{(p h)}$ do not manifest quantum fluctuations at all.

Let us now establish a contact with the definitions of AM given by Eqs. (13) and (14). Consider first the spin density operator

$$
\vec{S}(\vec{r})=\frac{1}{4 \pi c} \vec{E}(\vec{r}) \times \vec{A}(\vec{r})
$$

in the case of E1 monochromatic radiation. Using (10), one can see that the components of (23) contain all possible bilinear combinations of photon operators (11). Taking into account the property of spherical Bessel functions that $j_{0}(k r) \rightarrow 1$ and $j_{2}(k r) \rightarrow 0$ at $r \rightarrow 0$, we can conclude that the components of the spin operator (23) have the same structure in the photon operators as (21). Moreover, it is seen that the integrand of the second term in (14) vanish in the same limit. Thus, in a certain vicinity of the origin (atom), AM of photons consists of spin, while OAM contribution arises with distance from the source.

Taking into account that the photon localization appears in the form of a wavefront [29], we should integrate (23) over a spherical shall of radius $r$ together with averaging over time to calculate the amount of spin carried by E1 photon at any distance $r$ from the source. Performing straightforward but tedious calculations, we can conclude that

$$
\begin{array}{r}
\overrightarrow{\mathcal{S}}(r) \equiv \int_{0}^{4 \pi} d \phi \int_{0}^{\pi} \vec{S}(\vec{r}) \sin \theta d \theta=f(k r) \vec{J}^{(p h)}, \\
f(k r) \sim \frac{1}{3}\left[2 j_{0}^{2}(k r)-\frac{1}{2} j_{2}^{2}(k r)\right] .
\end{array}
$$

In turn, OAM of E1 photons at distance $r$ from the source can be calculated in the same fashion as $\overrightarrow{\mathcal{S}}(r)$ :

$$
\overrightarrow{\mathcal{L}}(r) \equiv \int_{0}^{4 \pi} d \phi \int_{0}^{\pi} \vec{L}(\vec{r}) \sin \theta d \theta \sim \frac{1}{2} j_{2}^{2}(k r) \vec{j},
$$


so that

$$
\int_{0}^{R}\left[f_{S}(k r)+f_{L}(k r)\right] r^{2} d r=1
$$

as all one can expect.

The fact that OAM has the same operator structure as the spin and total AM reflects the known property of electric-type photons $[3,25]$. Viz, in the states described by the vector spherical harmonics of electric type (8), OAM does not have a given value but is a superposition of states with $\ell=j \pm 1$. Thus, in these states, the total AM cannot be divided into spin and OAM contributions.

A more detailed examination shows that, unlike the energy of electromagnetic field, AM is not contained in the pure wave zone, and the main contribution to AM comes from the near and intermediate zones. At far distances where

$$
j_{\ell}(x) \sim[\sin (x-\ell \pi / 2)] / x, \quad x \gg \ell,
$$

we get $\vec{S}(k r)=\vec{L}(k r)$, so that the spin and OAM contribute equally into the total AM at far distances.

Consider now the radiation by atom in free space, when the continuum mode distribution, corresponding to the natural line breadth, should be taken into account [17]. In this case, we should extend the model Hamiltonian Hamiltonian (17) on the multi-mode case by adding integration over $k$ and apply the Markov approximation Markov approximation, which is similar to the WignerWeisskopf approach [30,31]. Then, the time-dependent wave function of the atom-photon system can be written in the form

$$
|\psi(t)\rangle=C(t)\left|\psi^{(a)}\right\rangle+\int B(k, t)|\psi(k)\rangle d k
$$

with the initial conditions

$$
C(0)=1, \quad \forall k \quad B(k, 0)=0 .
$$

Here $\left|\psi^{(a)}\right\rangle$ corresponds to the excited atomic state and vacuum for photons, while $|\psi(k)\rangle$ gives the ground atomic state and single E1 photon with given $k$ and $m$. Employing the standard analysis $[30,31]$ than gives

$$
C(t)=e^{-i \omega_{0} t-\Gamma t}, \quad B(k, t)=\frac{-k^{3 / 2}}{\omega_{k}-\omega_{0}+i \Gamma}\left(1-e^{i\left(\omega_{k}-\omega_{0}\right)-\Gamma t}\right)
$$

where $\Gamma$ is the radiative decay width.

Carrying out the averaging of $z$ components of spin and OAM contributions in (14) over the state (24), we get [17]

$$
\left\langle S_{z}(t)\right\rangle=\left\langle L_{z}(t)\right\rangle=\frac{1}{2}\left(1-e^{-2 \Gamma t}\right) .
$$


Since the Markov approximation Markov approximation corresponds to the "rough" scale $t \gg \Gamma^{-1}$ [31], Eq. (25) shows that spin and OAM contribute equally into the total $\mathrm{AM}$ of $\mathrm{E} 1$ photons at the distances $r \geq c / \Gamma \gg c / \omega_{0}$, again corresponding to the wave zone.

The obtained results can also be applied to the problem of entanglement of photon twins created by an electric quadrupole (E2) transition between the states $|j=2, m=0\rangle$ and $\left|j^{\prime}=0, m^{\prime}=0\right\rangle$ (Fig. 2) [17]. The cascade decay of this state gives rise to the two E1 photons propagating in the opposite directions. Because of the AM conservation, the state of the radiation field has the form

$$
|\psi\rangle=\frac{1}{\sqrt{3}}\left(\left|1_{+}, 1_{-}\right\rangle+\left|1_{-}, 1_{+}\right\rangle+\left|1_{0}, 1_{0}\right\rangle\right)
$$

where the subscripts correspond to the quantum numbers $m$ and $\left|1_{m}, 1_{m^{\prime}}\right\rangle$ is the product of number states of "left" and "right" photons. Let us stress that photons with $m=0$ may have the most probable direction of propagation different from that for the photons with $m= \pm 1$ because of the structure of the radiation pattern.

We now prove that (26) represents the maximum entangled qutrit state. It was shown in Refs. $[32,33]$ that the maximum entangled states entangled states of a composite system obey the following criterion. Local measurement The local measurements at all subsystems have maximum uncertainty in comparison with the other states allowed for a system under consideration. The complete set of local measurements is defined by the dynamic symmetry group of the Hilbert state of the composite system [33]. In the case of qutrit system, this is the $S U(3)$ group. Then, the local ("left" and "right") measurements in the system under consideration are described by the nine Hermitian generators of the $S U(3)$ subalgebra in the Weil-Heisenberg algebra of E1 photons (11):

$$
M=\left\{\begin{array}{ccc}
a_{+}^{+} a_{+}-a_{0}^{+} a_{0}, & a_{0}^{+} a_{0}-a_{-}^{+} a_{-}, & a_{-}^{+} a_{-}-a_{+}^{+} a_{+}, \\
\frac{1}{2}\left(a_{+}^{+} a_{0}+a_{0}^{+} a_{+}\right), & \frac{1}{2}\left(a_{0}^{+} a_{-}+a_{-}^{+} a_{0}\right), & \frac{1}{2}\left(a_{-}^{+} a_{+}+a_{+}^{+} a_{-}\right), \\
\frac{1}{2 i}\left(a_{+}^{+} a_{0}-a_{0}^{+} a_{+}\right), & \frac{1}{2 i}\left(a_{0}^{+} a_{-}-a_{-}^{+} a_{0}\right), & \frac{1}{2 i}\left(a_{-}^{+} a_{+}-a_{+}^{+} a_{-}\right) .
\end{array}\right.
$$

It is easily seen that

$$
\left\langle\psi\left|M_{n}\right| \psi\right\rangle=0
$$

for all $n=1, \cdots, 9$ in (27). Thus, the uncertainties of the measurements (27)

$$
\left\langle\left(\Delta M_{n}\right)^{2}\right\rangle \equiv\left\langle\left(M_{n}\right)^{2}\right\rangle-\left\langle M_{n}\right\rangle^{2}
$$

achieve the maximum value $\left\langle\left(\Delta M_{n}\right)^{2}\right\rangle=\left\langle\left(M_{n}\right)^{2}\right\rangle$ in the case of averaging over the state (26).

Let us stress that similar qutrit states have been considered in the context of quantum information processing and quantum cryptography $[34,35]$. 
It is easily seen that AM operators (22) can be constructed as the linear combinations of the generators (27). Thus, $\left\langle J_{\alpha}^{(p h)}\right\rangle=0$ in the state (26). At the same time, this state provide the maximum quantum fluctuations of the components of AM

$$
\left\langle\left(\Delta J_{\alpha}^{(p h)}\right)^{2}\right\rangle=\frac{2}{3}, \quad \alpha=x, y, z,
$$

as well as the maximum correlation of measurements at the opposite sides of the "quantum information channel" provided by the state (26):

$$
\left\langle\left[J_{\alpha}^{(p h)}\right]_{\text {left }},\left[J_{\alpha}^{(p h)}\right]_{\text {right }}\right\rangle=\frac{2}{3} .
$$

Here $\langle A, B\rangle \equiv\langle A B\rangle-\langle A\rangle\langle B\rangle$. This results illustrates the idea that the entangled states entangled states carry information in the form of correlations between the local measurements Local measurement [36].

\section{Quantum Phase of E1 Photons}

The problem of quantum phase was discussed in quantum optics for a long time (for review, see Refs. [37, 38, 39]). Among the results in the field, the two should be mentioned, first of all. One is the so-called Pegg-Barnett approach $[40,41]$ (for further references, see [38]). Their method is based on a contraction of the infinite-dimensional Hilbert space of photons $\mathcal{H}$. Viz, the quantum phase phase is first defined in an arbitrary $s$-dimensional subspace in $\mathcal{H}$. The formal limit $s \rightarrow \infty$ is taken only after the averaging of the operators, describing the physical quantities. A weak spot of the approach is that any restriction of dimension of the Hilbert-Fock space Fock space of photons leads to an effective violation of the algebraic properties of the photon operators. This, in turn, can lead to an inadequate picture of quantum fluctuations.

Another approach has been proposed by Noh, Fougères, and Mandel [42, 43]. It is based on the operational definition of the quantum phase (in terms of what can be measured). The main result of the approach is that there is no unique quantum phase variable, describing universally the measured phase properties of the light. This very strong statement has obtained a totally convincing confirmation in a number of experiments.

The use of SWR permits us to define the quantum phase phase of photons, corresponding to the azimuthal phase of AM $[18,21]$, in the whole Hilbert space without any contraction. The approach proposed in Ref. [18] complements, in a sense, the Noh-Fougères-Mandel approach. In fact, it defines the quantum phase in terms of what can be emitted by a source.

Let us use again the Jaynes-Cummings Hamiltonian (18) and the atomic AM (20). The latter can be specified by the operators

$$
J_{+}^{(a)}=\sqrt{2}\left(R_{+0}+R_{0-}\right), \quad J_{-}^{(a)}=\left(J_{+}^{(a)}\right)^{+}, \quad J_{z}^{(a)}=R_{++}-R_{--},
$$


forming a representation of the $S U(2)$ algebra in the three-dimensional Hilbert space of excited atomic states:

$$
\left[J_{+}^{(a)}, J_{-}^{(a)}\right]=2 J_{z}^{(a)}, \quad\left[J_{z}^{(a)}, J_{ \pm}^{(a)}\right]= \pm J_{ \pm}^{(a)} .
$$

Since the enveloping algebra of (28)-(29) contains the uniquely defined Casimir operator

$$
\left(\vec{J}^{(a)}\right)^{2}=2 \sum_{m=-1}^{1} R_{m m}=2 \times \mathbf{1},
$$

where 1 denotes the unit operator in the three-dimensional Hilbert space, a dual phase-dependent representation of (28) can be constructed through the use of method proposed by Vourdas [44]. Viz, the rising and lowering operators in (28) can be represented in the "polar" form

$$
J_{+}^{(a)}=J_{r}^{(a)} E, \quad J_{-}^{(a)}=E^{+} J_{r}^{(a)},
$$

where $J_{r}^{(a)}$ is the Hermitian "radial" operator and $E$ is the unitary $\left(E E^{+}=1\right)$ "exponential of the phase" operator. It is easily seen that

$$
E=R_{+0}+R_{0-}+e^{i \psi} R_{-+},
$$

where $\psi$ denotes an arbitrary real reference phase. Using (30), one can define the cosine and sine of the atomic AM azimuthal phase operators

$$
C^{(a)}=\frac{1}{2}\left(E+E^{+}\right), \quad S^{(a)}=\frac{1}{2 i}\left(E-E^{+}\right),
$$

such that

$$
\left[C^{(a)}, S^{(a)}\right]=0 \quad \text { and } \quad\left(C^{(a)}\right)^{2}+\left(S^{(a)}\right)^{2}=1 .
$$

The phase states of the atomic AM are then defined to be the eigenstates of the operator (30)

$$
E\left|\phi_{m}\right\rangle=e^{i \phi_{m}}\left|\phi_{m}\right\rangle
$$

which leads

$$
\left|\phi_{m}\right\rangle=\frac{1}{\sqrt{3}} \sum_{m^{\prime}=-1}^{1} e^{-i m^{\prime} \phi_{m}}|j=1, m\rangle, \quad \phi_{m}=\frac{\psi+2 m \pi}{3},
$$

where $m$ acquires the values 0 and \pm 1 as above. Through the use of the phase states (32), it is easy to define the following dual representation of the $S U(2)$ algebra (28)-(29):

$$
\mathcal{J}_{ \pm}^{(a)}=\sum_{m} \sqrt{2-m(m \pm 1)}\left|\phi_{m \pm 1}\right\rangle\left\langle\phi_{m}\left|, \quad \mathcal{J}_{z}^{(a)}=\sum_{m} m\right| \phi_{m}\right\rangle\left\langle\phi_{m}\right| .
$$


It should be stressed that the $S U(2)$ phase states can be constructed for an arbitrary number of two-level atoms. In particular, it can be shown that the $S U(2)$ phase states form the set of maximum entangled $2 N$-qubit states [32].

The representation of the $S U(2)$ subalgebra in the Weyl-Heisenberg algebra of E1 photons (11) has the form

$J_{+}^{(p h)}=\sqrt{2}\left(a_{+}^{+} a_{0}+a_{0}^{+} a_{-}\right), \quad J_{-}^{(p h)}=\left(J_{+}^{(p h)}\right)^{+}, \quad J_{z}^{(p h)}=\sum_{m} m a_{m}^{+} a_{m}$.

This expressions can be obtained directly from (22). The operators (34) complement the atomic operators (28) with respect to an integral of motion with the Hamiltonian (18). Unfortunately, there is no isotype representation of the $S U(2)$ subalgebra in the Weyl-Heisenberg algebra [45]. In other words, there is no uniquely defined Casimir operator in the enveloping algebra of (34). Therefore, Vourdas' [44] approach cannot be directly used here to describe the phase properties of $\mathrm{AM}$ of $\mathrm{E} 1$ photons.

At the same time, we again can use the conservation of AM in the process of radiation, which is independent of whether we use the standard form of AM operators or their dual representation. In particular, it is seen that [18]

$$
[(E+\varepsilon), H]=0,
$$

where

$$
\varepsilon=a_{+}^{+} a_{0}+a_{0}^{+} a_{-}+e^{i \psi} a_{-}^{+} a_{+}
$$

is the photon counterpart of the exponential of the phase operator (30). In contrast to (30), Eq. (35) does not determine a unitary operator. At the same time, (35) represents the normal operator

$$
\left[\varepsilon, \varepsilon^{+}\right]=0
$$

commuting with the total number of photons

$$
\left[\varepsilon, \sum_{m} a_{m}^{+} a_{m}\right]=0
$$

The quantum phase phase properties of E1 photons can now be described in terms of the dual representation of photon operators that has been introduced in Ref. [21]. Let us use the following Bogolubov-type [46] canonical transformation

$$
a_{m}=\frac{1}{\sqrt{3}} \sum_{m^{\prime}=-1}^{1} e^{-i m^{\prime} \phi_{m}} \mathbf{a}_{m^{\prime}}, \mathbf{a}_{m}=\frac{1}{\sqrt{3}} \sum_{m^{\prime}=-1}^{1} e^{i m^{\prime} \phi_{m}} a_{m^{\prime}},\left[\mathbf{a}_{m}, \mathbf{a}_{m^{\prime}}^{+}\right]=\delta_{m m^{\prime}} \cdot(39)
$$


Here $\phi_{m}$ represents the same phase angle as above. It is seen that the operator (35) takes the diagonal form in the representation (36):

$$
\varepsilon_{\phi}=\sum_{m=-1}^{1} e^{i \phi_{m}} \mathbf{a}_{m}^{+} \mathbf{a}_{m} .
$$

Let us note that the atomic operator (30) is also diagonal in the representation of phase states (32)

$$
E_{\phi}=\sum_{m} e^{i \phi_{m}}\left|\phi_{m}\right\rangle\left\langle\phi_{m}\right|
$$

and that

$$
\left[\left(E_{\phi}+\varepsilon_{\phi}\right), H\right]=0 .
$$

Thus, all one can conclude is that the operators $\mathbf{a}_{m}$ and $\mathbf{a}_{m}^{+}$(36) provide a representation of E1 photon operators of annihilation and creation with given quantum phase that, by construction, is the azimuthal phase of AM of photons.

In particular, the annihilation operators in the phase representation (36) obey the stability condition

$$
\forall m \quad \mathbf{a}_{m}|0\rangle=0,
$$

where $|0\rangle$ is the vacuum state. Thus, the conjugated creation operator can be used to construct the Fock number states in the phase representation in usual way:

$$
\left|\nu_{m}\right\rangle=\frac{1}{\sqrt{\nu_{m}}}\left(\mathbf{a}_{m}^{+}\right)^{\nu_{m}}|0\rangle, \quad \mathbf{a}_{m}^{+} \mathbf{a}_{m}\left|\nu_{m}\right\rangle=\nu_{m}\left|\nu_{m}\right\rangle, \quad \nu_{m}=0,1, \cdots,
$$

such that

$$
\left\langle\nu_{m} \mid \nu_{m^{\prime}}\right\rangle=\delta_{m m^{\prime}}, \quad \bigotimes_{m=-1}^{1} \sum_{\nu_{m}}\left|\nu_{m}\right\rangle\left\langle\nu_{m}\right|=1 .
$$

Thus, the photon phase states $\left|\nu_{m}\right\rangle$ form a complete orthonormal denumerable set of states of E1 photons, spanning the "phase" Hilbert-Fock space Fock space. This space is dual to the conventional space of states of E1 photons. It is seen that

$$
\varepsilon_{\phi}\left|\nu_{m}\right\rangle=\nu_{m} e^{i \phi_{m}}\left|\nu_{m}\right\rangle .
$$

Therefore, (37) can be interpreted as the non-normalized exponential of the phase operator. In turn, the cosine and sine of the photon phase phase operators can be defined as follows

$$
C_{\phi}^{(p h)}=K \sum_{m} \mathbf{a}_{m}^{+} \mathbf{a}_{m} \cos \phi_{m}, \quad S_{\phi}^{(p h)}=K \sum_{m} \mathbf{a}_{m}^{+} \mathbf{a}_{m} \sin \phi_{m},
$$


where the normalization coefficient $K$ is determined by the condition

$$
\left\langle\left(C_{\phi}^{(p h)}\right)^{2}+\left(S_{\phi}^{(p h)}\right)^{2}\right\rangle=1
$$

for the averaging over an arbitrary state of the radiation field. Similar coefficient was used in the Noh-Fougères-Mandel operational approach as well [43].

The phase representation (36) can be used to describe the azimuthal phase of AM of E1 photons [18, 19, 20, 21, 39]. In particular, it is possible to show that the eigenvalues of the azimuthal quantum phase of AM of photons have a discrete spectrum, depending on the number of photons. All eigenvalues lie in the interval $[0,2 \pi]$. In the classical limit, provided by the high-intensity coherent state coherent state of radiation, the phase eigenvalues are distributed uniformly over the interval $[0,2 \pi]$ as all one can expect in classical domain $[21,39]$.

The comparison with the Pegg-Barnett approach shows the qualitative coincidence of results for mean value of the cosine and sine operators. At the same time, there is a striking difference in the behavior of variance of quantum phase phase in the case of very few photons, corresponding to the quantum domain $[19,39]$.

\section{Polarization of Multipole Photons}

The polarization is usually defined to be the measure of transversal anisotropy of electromagnetic field with respect to the direction of propagation provided by the Poynting vector $\vec{P}$ [26]. Quantum electrodynamics interprets the polarization as given spin state of photons [3]. In spite of the fact that spin spin is equal to 1 and hence has three states, the photons have only two polarizations because of the absence of the rest mass.

In PWR, direction of $\vec{P}$ always coincides with $\vec{k} / k$, and the polarization is uniquely defined everywhere. However, this is no longer a case for SWR, where $(\vec{r} \times \vec{P})$ is not necessary equal to zero, at least in a certain vicinity of the source.

As a matter of fact, E1 radiation obey the condition $(\vec{r} \cdot \vec{B})=0$, while the electric field $\vec{E}$ is not orthogonal to the radial direction [2]. Therefore, if we consider the radiation in the "laboratory frame" spanned by the basis (4) with the origin at the atom location, the three polarizations should be taken into account $[22,23]$.

This fact can be illustrated in the following way. Within the relativistic picture, the field is described by the field-strength tensor

$$
F_{\alpha \beta}=\partial_{\alpha} A_{\beta}-\partial_{\beta} A_{\alpha}=\left(\begin{array}{cccc}
0 & E_{x} & E_{y} & E_{z} \\
-E_{x} & 0 & -B_{z} & B_{y} \\
-E_{y} & B_{z} & 0-B_{x} & \\
-E_{z} & -B_{y} & B_{x} & 0
\end{array}\right) .
$$


Since the anisotropy of the field is specified by the magnitudes of the components and by the phase angles between the components, consider the following $(4 \times 4)$ matrix

$$
\mathcal{R}=F^{+} F=\left(\begin{array}{cc}
\left(\vec{E}^{+} \cdot \vec{E}\right) & \vec{P} \\
\vec{P}^{+} & \mathcal{P}
\end{array}\right) .
$$

Here $F\left(F^{+}\right)$denotes the positive- (negative)-frequency part of (42), $\vec{P}$, apart from an unimportant factor, coincides with the positive-frequency part of the Poynting vector, and $\mathcal{P}$ is the Hermitian $(3 \times 3)$ matrix additive with respect to contributions coming from electric and magnetic fields

$$
\mathcal{P}=\mathcal{P}_{E}+\mathcal{P}_{B},
$$

where

$$
\mathcal{P}_{E \alpha \beta}=E_{\alpha}^{+} E_{\beta}, \quad \alpha, \beta=x, y, z
$$

and

$$
\mathcal{P}_{B \alpha \beta}=\left\{\begin{array}{ll}
\vec{B}^{+} \cdot \vec{B}-B_{\alpha}^{+} B_{\alpha} & \text { at } \alpha=\beta \\
-B_{\alpha}^{+} B_{\beta} & \text { otherwise }
\end{array} .\right.
$$

Thus, the matrix (43)-(45) specifies the magnitudes of the components and the phase angles between the components of the complex field strengths in the "laboratory frame" with the origin at the source location. We chose to interpret (43) as the general polarization polarization matrix.

To justify this choice, consider first the case of plane waves propagating in the $z$-direction. Then, because of the relations $B_{x}=-E_{y}$ and $B_{y}=E_{x}$, both terms in (43) are reduced to the same $(2 \times 2)$ matrix of the form

$$
\left(\begin{array}{cc}
E_{x}^{+} E_{x} & E_{x}^{+} E_{y} \\
E_{y}^{+} E_{x} & E_{y}^{+} E_{y}
\end{array}\right)
$$

that is, to the conventional polarization matrix [26]. In the case of multipole radiation, the matrices (44) and (45) can also be reduced to the $(2 \times 2)$ conventional polarization matrices by a local unitary transformation, rotating the $z$-axis in the direction of Poynting vector $\vec{P}(\vec{r})$ at any point $\vec{r}$.

The diagonal terms in (44) give the radiation intensities of the components. The off-diagonal terms give the "phase information" described by the phase differences

$$
\Delta_{\alpha \beta}=\arg E_{\alpha}-\arg E_{\beta}, \quad \Delta_{x y}+\Delta_{y z}+\Delta_{z x}=0 .
$$

The polarization matrices (44) and (45) can also be expressed in the helicity basis (4) through the use of the unitary transformation

$$
U\left(\begin{array}{c}
E_{x} \\
E_{y} \\
E_{z}
\end{array}\right)=\left(\begin{array}{c}
E_{+} \\
E_{0} \\
E_{-}
\end{array}\right), \quad U=\frac{1}{\sqrt{2}}\left(\begin{array}{ccc}
1 & i & 0 \\
0 & 0 & \sqrt{2} \\
-1 & i & 0
\end{array}\right)
$$


and similar transformation for $\vec{B}$. Then, $U \mathcal{P}_{E} U^{+}$coincides, to within the transposition of columns, with the polarization matrix with elements

$$
\tilde{\mathcal{P}}_{\mu \mu^{\prime}}=E_{\mu}^{+} E_{\mu^{\prime}}, \quad \mu, \mu^{\prime}=0, \pm 1,
$$

have been introduces in [22] for E1 radiation.

Since $\vec{E}(\vec{r}) \cdot \vec{B}(\vec{r})$ at any point $\vec{r}$ and $\vec{r} \cdot \vec{B}=0$ for the electric-type radiation, the complete information about the phase differences is provided by the matrix (44) or by the equivalent matrix (46) in this case. In the case of magnetic-type radiation with $\vec{r} \cdot \vec{E}=0$, the matrix (45) should be used instead of (44) [47].

Consider now the quantum E1 radiation. In this case, the field amplitudes should be changed by corresponding operators. Let us note that, in addition to (46), defined in terms of normal product of photon operators, we can also construct the anti-normal ordered polarization matrix

$$
\tilde{\mathcal{P}}_{\mu \mu^{\prime}}^{(a n)}=E_{\mu^{\prime}} E_{\mu}^{+} .
$$

Then, the difference

$$
\tilde{\mathcal{P}}_{\mu \mu^{\prime}}^{(0)} \equiv \tilde{\mathcal{P}}_{\mu \mu^{\prime}}^{(a n)}-\tilde{\mathcal{P}}_{\mu \mu^{\prime}}=\left[E_{\mu}, E_{\mu^{\prime}}^{+}\right]
$$

defines the elements of the vacuum polarization matrix, in other words, the zero-point oscillations (ZPO) of polarization [48]. It is easily seen that ZPO of polarization depend on the distance from the source $r$ and have a uniform angular distribution. Consider first the $z$-direction when $\theta=0$ and

$$
Y_{j \pm 1, m-\mu}(0, \phi)=\sqrt{\frac{2(j \pm 1)+1}{4 \pi}} \delta_{m \mu}
$$

for all $\phi$. Then, taking into account the definition of vector spherical harmonics (5), operator vector potential (10), and commutation relations (11), for the right-hand side of (47) we get

$\tilde{\mathcal{P}}_{\mu \mu^{\prime}}^{(0)}(r, 0, \phi)= \begin{cases}N_{E}^{2}\left[j_{2}(k r)\langle 12 \mu 0 \mid 1 \mu\rangle \sqrt{\frac{5}{4 \pi}}-j_{0}(k r) \sqrt{\frac{1}{2 \pi}}\right]^{2}, & \text { at } \mu=\mu^{\prime} \\ 0, & \text { otherwise }\end{cases}$

Because of the $S U(2)$ invariance of the operator vector potential, proven in Sec. II, there is a local unitary transformation $V(\vec{r})$, transforming (47) into (48) at any point. For explicit form of $V$ see Ref. [39]. Since $\langle 1210 \mid 11\rangle=$ $\langle 12,-1,0 \mid 1,-1\rangle=1 / \sqrt{10}$ and $\langle 1200 \mid 10\rangle=-\sqrt{(2 / 5)}$, the transversal (with respect to $\vec{r}$ ) elements $\tilde{\mathcal{P}}_{ \pm \pm}^{(0)}$ in (48) have equal magnitude. In view of definition of spherical Bessel functions, it is seen that ZPO of polarization are strong enough in the near and intermediate zones, while vanish at $r \rightarrow \infty$. 
Let us now apply the above unitary transformation to the components of the operator vector potential of E1 field $V(\vec{r}) \vec{A}_{E 1 k \mu}(\vec{r})$ and calculate the commutator

$$
\left[V(\vec{r}) \vec{A}_{E 1 k \mu}(\vec{r}), \vec{A}_{E 1 k \mu^{\prime}}^{+}(\vec{r}) V^{+}(\vec{r})\right]=\delta_{\mu \mu^{\prime}} \times \begin{cases}\tilde{\mathcal{P}}_{++}^{(0)}(r) & \text { at } \mu= \pm 1 \\ \tilde{\mathcal{P}}_{00}^{(0)}(r) & \text { at } \mu=0\end{cases}
$$

It is seen that these relations coincide with (11) to within the distance-dependent factors, describing ZPO of polarization. In turn, the normalized operators

$$
b_{k \mu}(\vec{r})=\frac{V(\vec{r}) \vec{A}_{E 1 k \mu}(\vec{r})}{\sqrt{\tilde{\mathcal{P}}_{\mu \mu}^{(0)}(r)}}
$$

obey the commutation relations (11) at any point $\vec{r}$ and hence form a local representation of the Weyl-Heisenberg algebra of E1 photons. Instead of the global index $m$, specifying AM, they depend on the coordinates $\mu$. In other words, they specify the field oscillations in the "laboratory frame" spanned by the helicity basis (4) and hence can be interpreted as the local operators of EI photons with given polarization [48]. This means that the eigenstates of the number operators $b_{\mu}^{+}(\vec{r}) b_{\mu}(\vec{r})$ give the number states of E1 photons with given polarization $\mu$ at any point $\vec{r}$.

In fact, Eq. (49) represents a local Bogolubov-type canonical transformation from the photon operators with given $m$ to the photon operators with given $\mu$

$$
b_{k \mu}(\vec{r})=\frac{1}{\sqrt{\tilde{\mathcal{P}}_{\mu \mu}^{(0)}(r)}} \sum_{m} \sum_{\mu^{\prime}}(-1)^{\mu^{\prime}} V_{\mu \mu^{\prime}}(\vec{r}) \mathcal{A}_{k m \mu^{\prime}}(\vec{r}) a_{m} \equiv \sum_{m} \mathcal{B}_{m \mu}(\vec{r}) a_{m}
$$

where $\mathcal{A}$ denotes the mode function (see Sec. II). Since $\operatorname{det}[\mathcal{B}] \neq 0$, there is an inverse transformation, representing operators $a_{m}$ in terms of $b_{\mu}$.

It is a straightforward matter to show that, in the representation of local operators (49)-(50), the polarization matrix (46), apart from an unimportant constant factor, takes the form

$$
\tilde{\mathcal{P}}_{\mu \mu^{\prime}}(\tilde{r})=b_{\mu}^{+}(\vec{r}) b_{\mu^{\prime}}(\vec{r}) .
$$

Assume now that the two-level E1 transition have been discussed in Sec. III emits a single photon in the state $\left|1_{m}\right\rangle$. Then, the averaging of (51) over this state gives the polarization at any point $\vec{r}$ described by the matrix with elements

$$
\left\langle\tilde{\mathcal{P}}_{\mu \mu^{\prime}}(\vec{r})\right\rangle=\frac{\mathcal{B}_{m \mu}^{*}(\vec{r}) \mathcal{B}_{m \mu^{\prime}}(\vec{r})}{\tilde{\mathcal{P}}_{\mu \mu}^{(0)}(r)} .
$$

Taking again into account that the properties of a multipole photon correspond to a spherical shall of radius $r$, we should integrate (52) over $\sin \theta d \theta d \phi$ to get 
the polarization matrix of the photon at any distance $r$ from the atom like we did in Sec. III for the spin carried by photon. In particular, it can be seen that the photons with any $m$ have only two circular polarizations $\mu= \pm 1$ in the wave zone [23] even in the "laboratory frame".

It should be emphasized that the polarization can also be described in terms of Stokes parameters stokes parameters that become the Stokes operators in quantum domain [49]. Since in the "laboratory frame" we have three degrees of freedom, the set of Hermitian Stokes operators is provided by the generators of the $S U(3)$ subalgebra in the local Weyl-Heisenberg algebra of operators (49). In other words, the Stokes operators in "laboratory frame" coincide with (27) with the substitution of $b_{\mu}(\vec{r})$ instead of $a_{m}$ [39].

In this way, the quantum properties of polarization of E1 photons can be described, including the quantum fluctuations of polarization [23, 39].

\section{Summary}

We have reviewed some recent results concerning the quantum radiation by multipole transitions in atoms and molecules. It is shown that because of the difference in the dynamic symmetry group of the Hilbert space, the use of SWR leads to an adequate picture of AM in quantum domain, based on the structure of the type $S U(2) \times S U(2)$. In particular, SWR permits us to evaluate the quantum fluctuations of AM of photons. It is also shown that spin and OAM contribute equally into the total AM of photons in the wave zone, while spin prevail over OAM in the near and intermediate zones.

It is also shown that the cascade decay of E2 transition can lead to creation of E1 photon twins in the qutrit state. This state obey the criterion of maximum entanglement of Refs. [32,33] and manifests maximum correlation of local measurements of AM.

The use of SWR permits us to define the inherent quantum phase of multipole photons that is the azimuthal phase of AM. This definition develops the operational approach by Noh, Fougëres and Mandel [42, 43]. The approach based on the polar decomposition of AM does not violate the algebraic properties of photon operators and leads to a qualitatively different picture of quantum fluctuations of phase from that obtained within the Pegg-Barnett approach $[38,40,41]$.

It should be stressed that the approach based on the consideration of the $S U(2)$ phase states has shown its efficiency in the problem of definition of maximum entangled $\mathrm{N}$-qubit states in atomic systems [32] of the type proposed in Ref. [50, 51], and in quantum cryptography [52]. In particular, it can be used to specify the qubit multipartite states in three-level atoms [53]. It can also be 
applied to classification of maximum entangled states entangled states that can be obtained through the use of strong-driving-assisted processes in cavity QED [54].

Finally, the use of SWR permits us to describe the quantum properties of polarization at any distance from the source. Since the polarization is a local property of multipole radiation, the representation of the Weyl-Heisenberg algebra of photons with given polarization at any distance from the source can be constructed through the use of SWR. It should be stressed that usually the problem of photon localization is discussed in terms of wave functions (see [55] and references therein). At first sight, there is no principle contradiction between the approaches based on the use of operators and wave functions. A connection between the approaches deserves a more detailed investigation.

In fact, the most of results reviewed in this paper were obtained by mapping of the operators from the finite-dimensional atomic Hilbert space to the infinitedimensional Hilbert space of photons through the use of AM conservation. This procedure reflects an old idea that the properties of quantum radiation are specified by the source [56].

The SWR can be successfully used to describe the quantum properties of multipole radiation, generated by real atomic transitions. In many cases, the use of SWR permits us to avoid the difficulties peculiar to PWR. The approach based on the SWR has also manifested its efficiency in the number of problems connected with investigation of photon band-gap materials [57, 58].

Although the results discussed in this paper are mostly connected with E1 radiation, they can also be generalized on the case of an arbitrary quantum multipole radiation.

\section{References}

[1] Bateman, H. (1955). The Mathematical Analysis of Electric and Optical Wave-Motion. New York: Dover

[2] Jackson, J.D. (1975). Classical Electrodynamics. New York: Wiley.

[3] Berestetskii, V.B., E.M. Lifshitz, and L.P. Pitaevskii. (1982). Quantum Electrodynamics. Oxford: Pergamon Press.

[4] Cohen-Tannouji, C., J. Dupont-Roc, and G. Grinberg. (1992). Atom-Photon Interaction. New York: Wiley.

[5] Wigner, E.P. (1959). Group Theory and Its Application to the Quantum Mechanics of Atomic Spectra. New York: Academic Press.

[6] Heitler, W. (1954). The Quantum Theory of Radiation, 3rd edition. London: Clarendon Press.

[7] Heitler, W. (1936). Proc. Camb. Phil. Soc. 37, 112.

[8] Bederson, B. and H. Walther. (2000). Advances in Atomic, Molecular, and Optical Physics, Vol. 42. New York: Academic Press.

[9] Raymond, J.M., M. Brune, and S. Haroche. (2001). Rev. Mod. Phys. 73, 565. 
[10] Arnavut, H.H. and G.A. Barbosa. (2000). Phys. Rev. Lett. 85, 286.

[11] Mair, A., A. Vazari, G. Weihs, and A. Zeilinger. (2001). Nature 412, 313.

[12] Padgett, M.J., S.M. Barnett, S. Franke-Arnold, and J. Courtial. (2002). Phys. Rev. Lett. 88, 257901.

[13] Vazari, A., G. Weihs, and A. Zeilinger. (2002). Phys. Rev. Lett. 89, 240401.

[14] Muthukrishnan, A. and C.R. Stroud. (2002). J. Opt. B 4, S73.

[15] Mandel, L. and E. Wolf. (1995). Optical Coherence and Quantum Optics, New York: Cambridge University Press.

[16] Van Enk, S.J. and G. Nienhuis. (1994). J. Mod. Opt. 41, 963.

[17] Cakır, Ö. and A.S. Shumovsky. (2003). E-print quant-ph

[18] Shumovsky, A.S. (1997). Opt. Commun. 136, 219.

[19] Shumovsky, A.S. and Ö. Müstecaplı个 glu.(1998).Opt.Commun.146, 124

[20] Shumovsky, A.S. and Ö. Müstecaplıo $\uparrow$ glu. (1998). In Causality and Locality in Modern Physics, edited by G. Hunter, S. Jeffers, and J.-P. Vigier, Dordreht: Kluwer.

[21] Shumovsky A.S. (1999). J. Phys. A 32, 6589.

[22] Shumovsky, A.S. and Ö. Müstecaplı^ glu. (1998). Phys. Rev. Lett. 80, 1202.

[23] Can, M.A. and A.S. Shumovsky. (2002). J. Mod. Opt. 49, 1423.

[24] Allen, L., M.J. Padgett, and M. Babiker. (1999). In Progress in Optics XXXIX, edited by E. Wolf. New York: Elsevier Science.

[25] Davydov, A.S. (1976). Quantum Mechanics Oxford: Pergamon Press.

[26] Born, M. and E. Wolf. (1970). Principles of Optics Oxford: Pergamon Press.

[27] Thompson, W.J. (1994). Angular Momentum New York: Wiley.

[28] Klyachko, A.A. (2000). Private communication.

[29] Acharya, R. and E.C.G. Sudarshan. (1960). J. Math. Phys. 1, 532.

[30] Davies, B. (1972). Quantum Theory of Open Systems New York: Academic Press.

[31] Gardiner, C.W. and P. Zoller. (2000). Quantum Noise Berlin: Springer.

[32] Can, M.A., A.A. Klyachko, and A.S. Shumovsky. (2002). Phys. Rev. A 66, 022111.

[33] Klyachko, A.A. and A.S. Shumovsky. (2003), J. Opt. B 5 (in print); preprint quant-ph/0302008. 
[34] Burlakov, A.V., M.V. Chekhova, O.A. Karabutova, D.N. Klyshko, and S.P. Kulik. (1999). Phys. Rev. A 60, R4209.

[35] Bechmann-Pasquinucci, H. and A. Peres (2000). Phys. Rev. Lett. 85, 3313.

[36] Brukner, Č., M. Žukowski, and A. Zeilinger. (2001). E-print quant$\mathrm{ph} / 0106119$.

[37] Lynch, R. (1995). Phys. Rep. 256, 367.

[38] Tanaś, R., A. Miranowitz, and T. Gantsog. (1996). Prog. Opt. 35, 1.

[39] Shumovsky, A.S. (2001). In Modern Nonlinear Optics, Pat 1, Advances in Chemical Physics, Vol. 119, edited by M.W. Evans, Series editors I. Prigogine and S.A. Rice, New York: Wiley.

[40] Pegg, D.T. and S.M. Barnett. (1988). Europhys. Lett. 6, 483.

[41] Pegg, D.T. and S.M. Barnett. (1997). J. Mod. Opt. 44, 225.

[42] Noh, J.W., A. Fougères, and L. Mandel. (1991). Phys. Rev. Lett. 67, 1426.

[43] Noh, J.W., A. Fougères, and L. Mandel. (1992). Phys. Rev. A 45, 242.

[44] Vourdas, A. (1990). Phys. Rev. A, 41, 1653.

[45] Serre, J.-P. (1977). Linear Representations of Finite Groups New York: Springer.

[46] Bogolubov, N.N., A.M. Kurbatov, and A.S. Shumovsky (Eds.). (1991). N.N. Bogolubov Selected Works, Part II New York: Gordon and Breach.

[47] Wolf, E. (1954). Nuovo Cimento 12, 884.

[48] Klyachko, A.A. and A.S. Shumovsky. (2001). Laser Phys. 11, 57.

[49] Jauch, J.M. and F. Rohrlich. (1959). The Theory of Photons and Electrons Reading MA: Addison-Wesley.

[50] Plenio, M.B., S.F. Huelga, A. Beige, and P.L. Knight. (1999). Phys Rev. A 59, 2468.

[51] Beige, A., W.J. Munro, and P.L. Knight. (2000). Phys. Rev. A 62, 052102.

[52] Vourdas, A. (2002). Phys. Rev. A 64, 04321.

[53] Can, M.A., A.A. Klyachko, and A.S. Shumovsky. (2002). Appl. Phys. Lett. 81, 5072.

[54] Solano, E., G.S. Agarwal, and H. Walther. (2002). Preprint quant$\mathrm{ph} / 0202071$. 
[55] Can, K.W., C.K. Law, and J.H. Eberly. (2002). Phys. Rev. Lett. 88, 100402.

[56] Koo, I.C. and J.H. Eberly. (1976). Phys. Rev. A 14, 2174.

[57] Rupasov V.I. and M. Singh. (1996). Phys. Rev. Lett. 77, 338.

[58] John. S. and V.I. Rupasov. (1997). Phys. Rev. Lett. 79, 821. 